Modelling off Hugoniot Loading Using Ramp Compression in Single Crystal Copper

J. Hawreliak, B. A. Remington, H. Lorenzana, E. Bringa, J. Wark

January 18, 2011 
This document was prepared as an account of work sponsored by an agency of the United States government. Neither the United States government nor Lawrence Livermore National Security, LLC, nor any of their employees makes any warranty, expressed or implied, or assumes any legal liability or responsibility for the accuracy, completeness, or usefulness of any information, apparatus, product, or process disclosed, or represents that its use would not infringe privately owned rights. Reference herein to any specific commercial product, process, or service by trade name, trademark, manufacturer, or otherwise does not necessarily constitute or imply its endorsement, recommendation, or favoring by the United States government or Lawrence Livermore National Security, LLC. The views and opinions of authors expressed herein do not necessarily state or reflect those of the United States government or Lawrence Livermore National Security, LLC, and shall not be used for advertising or product endorsement purposes.

This work performed under the auspices of the U.S. Department of Energy by Lawrence Livermore National Laboratory under Contract DE-AC52-07NA27344. 


\title{
Modelling off Hugoniot Loading Using Ramp Compression in Single Crystal Copper.
}

\author{
J. Hawreliak and H.E. Lorenzana \\ Lawrence Livermore National Laboratory, Livermore, CA 94551, USA \\ E. M. Bringa \\ CONICET \& Instituto de Ciencias Básicas, \\ Universidad Nacional de Cuyo, Mendoza, 5500 Argentina
}

(Dated: December 13, 2010)

\begin{abstract}
The application of a ramp load to a sample is a method by which the thermodynamic variables of the high pressure state can be controlled. The faster the loading rate, the higher the entropy and higher the temperature. This paper describes moleculer dynamics (MD) simulations with 25 million atoms which investigate ramp loading of single crystal copper. The simulations followed the propagation of a 300ps ramp load to 3Mbar along the [100] direction copper. The simulations were long enough to allow the wave front to steepen into a shock, at which point the simulated copper sample shock melted.

PACS numbers:
\end{abstract}


All forms of laboratory compression experiments are dynamic, requiring a finite time for the external force to be applied. These timescales range from days is diamond anvil cells (DAC) to picoseconds in high pressure shock waves. DAC experiments, as the name suggests, compress a material between two diamond anvils. Varying the temperature in a DAC has allowed significant exploration of the pressure-temperature(PT) phase diagram of many materials [1]. While scientist continue to push the limit of the capabilities of this technique it will ultimately be constrained by the strength of the diamonds, which makes working above $350 \mathrm{GPa}$ very difficult. At the other temporal extreme shock compression applies an impulsive load to a sample forming a propagating discontinuity between two states of matter [2]. The actual thickness of the shock front is determined by the apparent material viscosity [3]. Shock compression experiments are basically inertially confined. In a shock wave pressure is maintained for the length of time it takes the shock front to propagate through the sample and reach a free surface from which the pressure is released. While this technique has provided some of the highest pressures achieved on record [4], due to conservation of energy and momentum at the shock front the thermodynamic states are restricted to a set defined by the Rankin Hugoniot jump conditions. This ultimately limits the usefulness of shock compression for studying ultra-high density solids as typical materials shocked above 300GPa will end up melting. Therefore, to achieve TPa pressures in a solid requires a lower entropy state then can be produced in a single shock. There has been interest in recent years in using ramp or multi-shock compression to achieve ultra-high pressure states of matter while keeping the temperature close to the isentrope [5]. In the high energy density physics regime, ramp or quasi-isentropic compression refers to a loading rate which would still be incredibly fast by DAC standards but is slow enough so that the thermodynamic end state does not reside on the Hugoniot [6]. The lure for scientists to these new dynamic compression techniques is the ability to achieve higher pressures and material densities is a solid then ever before in the laboratory [7]. Figure 1 shows a PT diagram for copper with the shock Hugoniot drawn. The labels on the shock Hugoniot refer to the Swegle-Grady strain rates associated with a steady shock [8]. The isentrope and isotherm loading paths are also drawn in Fig. 1. The interesting thing about using the ramp loading technique over a single shock is a shock can interograte a single point in parameter space while a ramp will allow the exploration of the infinite number of states that reside between the isentrope and Hugoniot. The grey region highlighted in Fig. 1 between the isentrope 


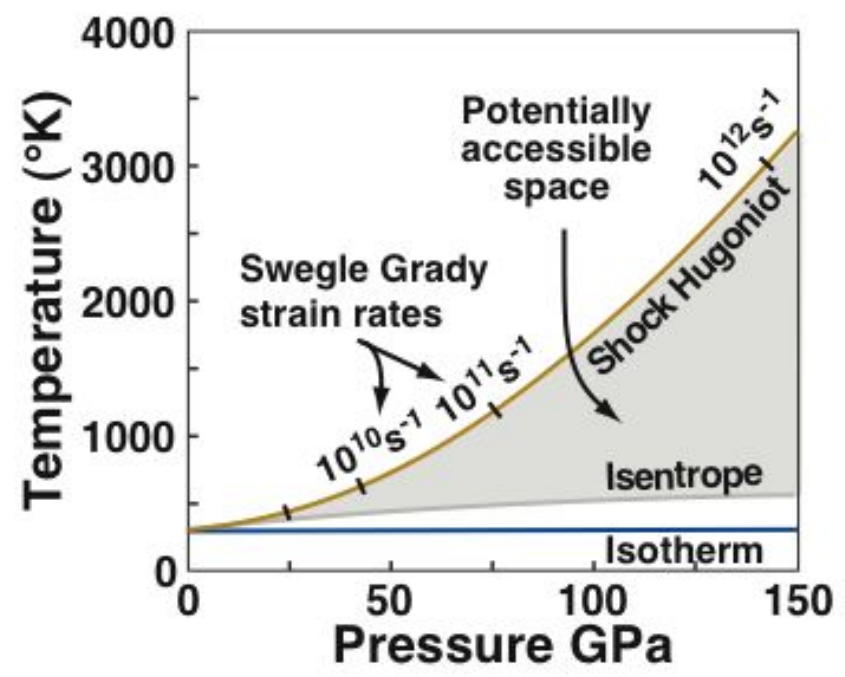

FIG. 1: A plot of pressure temperature states of the principle Hugoniot of copper, the loading isentrope of copper and an isotherm. The grey region between Hugoniot states and the isentrope are thermodynamic states that can be achieved through dynamic compression using different loading rates.

and Hugoniot are all the states accessible using ramp loading techniques. While in the limit the ramp can achieve isentropic compression, it will never be ideally isentropic as the there will be irreversible work and heat generated do to plasticity, the formation and motion of dislocations in the material. In this report we will show that a molecular dynamics (MD) simulation using a 300ps ramp we can achieve quasi-isentropically compression at the loading surface and as the wave propagates and the preceding shock strength increases we generate a series of states that fall between between the isentrope and the Hugoniot.

\section{RAMP COMPRESSION SIMULATIONS}

We have simulated the ramp compression of single crystal copper along the [100] direction with a peak particle velocity applied to the sample of $3.5 \mathrm{kms}^{-1}$, which corresponds to a peak pressure, in the shock case, of $250 \mathrm{GPa}$. This value was chosen as it lies above the shock-melt 
pressure for the copper Mishin potential[9], which previous studies have shown lies between 200 and $220 \mathrm{GPa}[10,11]$. While hydrodynamic simulations have been used to model ramp loading close to the isentrope, typical models of materials do not include microscopic effects like plastic work unless a strength model is used, and do not include the phase transition kinetics associated with melting. To generate a ramp compression in the MD simulation the velocity of a piston of atoms is increased linearly from 0 to $3.5 \mathrm{kms}^{-1}$, using $1003 \mathrm{psec}$ long discrete steps. After completing the ramp the peak velocity was held constant. While the loading technique was not a smooth linear ramp it is not incommensurate with experimental techniques used to achieve off-Hugoniot high pressures in dynamic compression experiments [12]. The use of multiple shocks or reverberating shocks is frequently used as a form of quasi-isnetropic compression because in the limit of small pressure changes the shock state approaches the isentrope.

$$
\Delta E=\lim _{V_{H}-V_{0} \rightarrow 0}^{I \rightarrow \infty} \sum_{i=0}^{I} \frac{\left(P_{H}+P_{0}\right)_{i}\left(V_{H}-V_{0}\right)_{i}}{2}=\int_{V_{0}}^{V} P(V) d V
$$

where $P_{H}$ and $P_{0}$ are the initial and shock pressure for the $i^{\text {th }}$ shock for $I$ total shocks, $V_{H}-V_{0}$ is the change in specific volume, and $\Delta E$ is the associated change in internal energy over the entire loading path. In the above equation the left hand side is the sequence of Rankine Hugoniot jump conditions associated with a series of shocks while the right hand is the isentrope, as the step size of the individual shocks get smaller these two become equal. The rise time of the ramp compare well with those used in recent shockless laser compression experiments. [13]. The size of the simulated sample was $25 \times 25 \times 10000$ conventional unit cells (25 million atoms), $4 \mu \mathrm{m}$ long along the compression direction, with periodic boundary conditions applied across the $(25 \times 25)$ width of the sample. The length of the sample was chosen to be long enough to allow the drive to reach full pressure and propagate until it steepened into a single shock. The initial temperature of the crystal was $10 \mathrm{~K}$, and was equilibrated before ramp compression. The equivalent strain rate for the material in contact with the piston is just above $10^{9} \mathrm{~s}^{-1}$.

In Fig. 2 we show the particle velocity and temperature as a function of depth within the sample. Profiles are shown for fourteen different times after the start of the ramp, ranging from 35 to 580 psec, at which point the compression wave is close to the end of the $4 \mu \mathrm{m}$ (original length) sample. The velocity snap shots show one important feature of ramp compression, the increase in the speed of sound due to the compression means the 
later portion of the ramp traveling into the compressed material will move faster speed then the foot of the ramp traveling into ambient material. This causes the compression to pile up at the leading edge and form a single shock. In our simulations after 115 psec a full elastic precursor forms, which maintains a constant amplitude of $0.6 \mathrm{kms}^{-1}$. For a perfect crystal along the [100] direction in copper using the Mishin potential this precursor corresponds to a pressure of about 30GPa. Proceeding the elastic shock is a slower plastic shock wave. There is very little heating due to the shock waves because the bulk of the high strain rate compression occurs in the elastic precursor, which by definition does no plastic work on the atoms. As the loading wave propagates the plastic shock wave increases in speed and intensity, until it over takes the the elastic precursor at about 280 ps after the start of the initial compression. Once the plastic shock has over taken the elastic precursor we see an increase in the temperature which continues to increases as the shock wave amplitude grows. The initial shock wave generates the majority of heating in the material with the proceeding ramp generating relatively little heat. This continues until the peak of the pressure wave overtakes the leading edge and there is only a single shock. Initially there is an over heating of the shock wave as it takes a finite time to nucleate the liquid phase and then melting can occur. Once melting can nucleate there is a drop in temperature associated with the latent heat of melting, $700 \mathrm{~K}$. The observation of the over driven solid phase is not observed in single shock MD simulations in Mishin copper where melting is initiated right at the piston interface. In the final timestep we see that the copper sample has a range of P-T states that cover from close to the isentrope to the shock melt state. While all ramp profiles will steepen to form a shock at a finite depth one could predict that a linear profile might not be the best to obtain the maximum volume of material at the lowest temperature due to the variation in sound speed with compression but it demonstrates in a single MD simulation the range of achievable states by adjusting the loading history. The temperature profiles in Fig 2 show that by tuning the initial shock strength the temperature of the end state can be selected. Depending on the material the pulse shape should start with a shallower slope allowing the low pressure compression to run further ahead before loading the higher pressure which can be done at a faster strain rate.

One important difference between shock loading and ramp loading is that in the shock case there is effectively a discontinuity between the low and high pressure state at the shock front. So the transition from the low to high pressure state is fast compared to the 


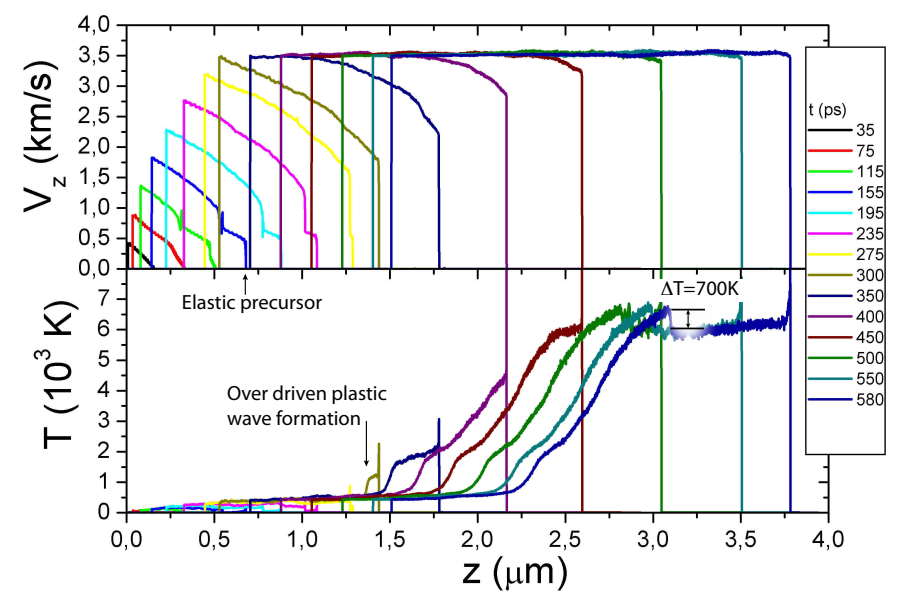

FIG. 2: (a) Velocity and (b) temperature profiles for different times during ramp loading of the $0.01 \times 0.01 \times 4 \mu \mathrm{m}^{3}$ sample. The linear ramp compression had a rise time of $300 \mathrm{ps}$ and a peak particle velocity of $3.5 \mathrm{kms}^{-1}$.

microscopic dynamics associated with the material response. After the shock front passes a particular location in the material it is the material dynamics which respond to the new higher pressure state. In the ramp case the material environment is changing while the material is continually responding to the applied strain. When the ramp is slow then the material state is effectively in equilibrium at any given point on the ramp. If the ramp is on a similar timescale as the material response timescale, which for the Mishin potential for copper is on the order of 10ps [11], then the material relaxation processes are competing with applied strain timescale . Figure 3 shows a section through the length of the MD simulation. The real space atoms are colored according to their calculated centro-symmetry parameter. The simulated diffraction, which is a slice through $\mathrm{x}, \mathrm{z}$ plane in reciprocal lattice space is calculated for discrete regions through out the simulation. The reciprocal lattice shows more dramatically the change in atomic ordering due to loading history. The region ahead of the loading front, Fig. 3d) shows the expected perfect crystal reciprocal lattice structure, with sharp peaks at each reciprocal lattice point. Looking at the diffraction closest to the piston Fig. 3a), where the sample is coldest upon compression, there is broadening of the diffraction spots in the [111] direction, which corresponds to stacking fault and twins responsible for the plastic deformation. In the region where the sample is shocked to a pressure of about 100GPa and then ramped compressed for the remaining 150GPa, we see a very interesting 

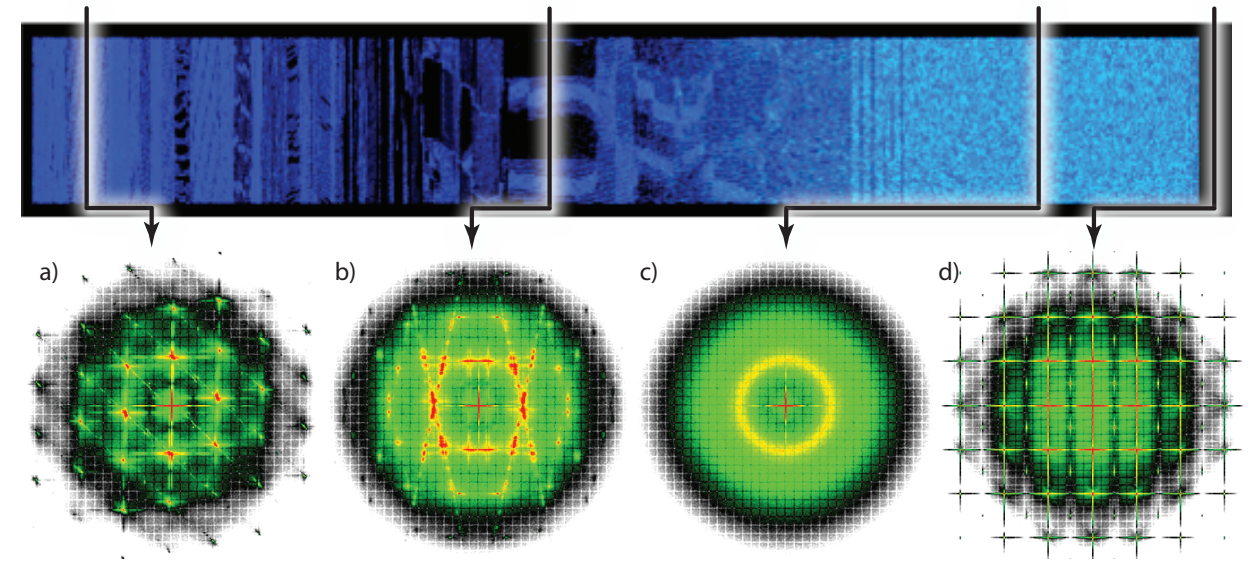

FIG. 3: real space image of the atoms whose centro-symmetry parameter suggests they are located in crystallie deffects. The difference in structure is made clear in the simulated x-ray diffraction images taken at a) close to the piston when the compression is nearly a continual ramp and the temperature remains cold, b) where there is a $1.5 \mathrm{MB}$ shock before a $1.5 \mathrm{MB}$ ramp and hold, c) where the loading profile has steepened to a point where it is shock melting and d) the virgin material before the loading wave has arrived.

deffect structure with dislocation plans forming along (100) planes slightly rotated from the compression direction. While the physicality of these defects is being investigated it is possibly due to the nature of the Mishin potential and that for perfect crystal the shock will compress the structure uniaxially to a point where it begins to Bain soften and the structure is more BCC in nature. When the ramp load has finally steepened up into a single shock, Fig. 3c), the crystalline structure is completely lost as can be seen by the liquid diffraction rings seen in the simulated x-ray diffraction.

The MD simulations shown here demonstrate that quasi-isentropic compression is possible using dynamic compression even at extremely high strain rates. A 300 ps ramp load applied to single crystal copper can maintain $2.5 \mu \mathrm{m}$ of solid material, with $1.5 \mu \mathrm{m}$ staying below 1000K. MD simulations like these can provide unique insight into the the material process which govern quasi-isnetropic compression. One particular area will be understanding the limiting strain rates associated with ramp compression. While continuum based hydrodynamic simulations are better suited for predicting the evolution of low strain rate loading paths at the higher pressures and strain rates the artificial viscosity used in hydro- 
dynamic simulations, to avoid discontinuities associated with shocks, will limit the ability to differentiate between a shock and a ramp. The MD simulations will be a key tool for examining the impact of different loading paths on microstructure as the microstructure determines material properties, such as strength and failure. [14].

\section{ACKNOWLEDGEMENTS}

The authors thank the Grand Computing Challenge at Lawrence Livermore National Laboratory whici provided the computer resources for the large scale computations. This work performed under the auspices of the U.S. Department of Energy by Lawrence Livermore National Laboratory under Contract DE-AC52-07NA27344 supported by the LDRD program Project No. 08-ER-033 at LLNL.

[1] A. Jayaraman, Rev. Mod. Phys. 55, 65 (1983).

[2] M. Meyers, Dynamic behavior of materials (Wiley-Interscience, 1994), ISBN 047158262X.

[3] Y. Zel'Dovich and Y. Raizer, Physics of shock waves and high-temperature hydrodynamic phenomena (Dover Pubns, 2002), ISBN 0486420027.

[4] R. Cauble, D. W. Phillion, T. J. Hoover, N. C. Holmes, J. D. Kilkenny, and R. W. Lee, Phys. Rev. Lett. 70, 2102 (1993).

[5] J. Davis, Journal of Applied Physics 99, 103512 (2006).

[6] K. Lorenz, M. Edwards, S. Glendinning, A. Jankowski, J. McNaney, S. Pollaine, and B. Remington, Physics of Plasmas 12, 056309 (2005).

[7] B. Remington, P. Allen, E. Bringa, J. Hawreliak, D. Ho, K. Lorenz, H. Lorenzana, J. Mcnaney, M. Meyers, S. Pollaine, et al., Materials Science and Technology 22, 474 (2006), ISSN 02670836 .

[8] J. Swegle and D. Grady, J. Appl. Phys 58, 603 (1985).

[9] Y. Mishin, M. Mehl, D. Papaconstantopoulos, A. Voter, and J. Kress, Physical Review B 63, 224106 (2001), ISSN 1550-235X.

[10] E. Bringa, J. Cazamias, P. Erhart, J. Stölken, N. Tanushev, B. Wirth, R. Rudd, and M. Caturla, Journal of Applied Physics 96, 3793 (2004). 
[11] E. Bringa, K. Rosolankova, R. Rudd, B. Remington, J. Wark, M. Duchaineau, D. Kalantar, J. Hawreliak, and J. Belak, Nature Materials 5, 805 (2006), ISSN 1476-1122.

[12] W. Nellis, Reports on Progress in Physics 69, 1479 (2006).

[13] J. Edwards, K. T. Lorenz, B. A. Remington, S. Pollaine, J. Colvin, D. Braun, B. F. Lasinski, D. Reisman, J. M. McNaney, J. A. Greenough, et al., Phys. Rev. Lett. 92, 075002 (2004).

[14] D. L. Preston, D. L. Tonks, and D. C. Wallace, Journal of Applied Physics 93, 211 (2002). 